\title{
Data Quality Objectives (DQO) for Solar Ultraviolet Radiation
}

Cite as: AIP Conference Proceedings 1100, 663 (2009); https://doi.org/10.1063/1.3117074

Published Online: 23 March 2009

Susana Diaz, Vitali Fioletov, Jay Herman, Liisa Jalkanen, Serm Janjai, Berit Kjeldstad, Takashi Koide, Gunther Seckmeyer, Paul Simon, Betsy Weatherhead, and Ann Webb

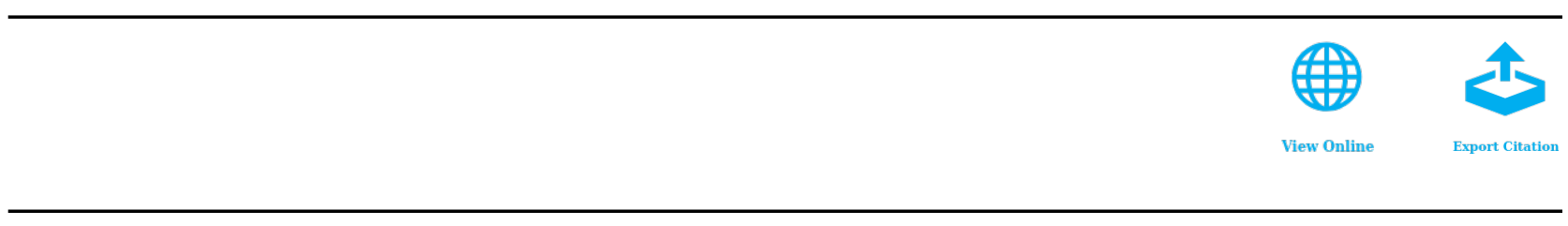




\title{
Data Quality Objectives (DQO) for Solar Ultraviolet Radiation
}

\author{
Susana Diaz ${ }^{\mathrm{a}}$, Vitali Fioletov ${ }^{\mathrm{b}}$ Jay Herman $^{\mathrm{c}}$, Liisa Jalkanen ${ }^{\mathrm{d}}$, Serm Janjai ${ }^{\mathrm{e}}$, \\ Berit Kjeldstad $^{\mathrm{f}}$, Takashi Koide ${ }^{\mathrm{g}}$, Gunther Seckmeyer ${ }^{\mathrm{h}}$, Paul Simon ${ }^{\mathrm{i}}$, Betsy \\ Weatherhead ${ }^{\mathrm{j}}$ and Ann Webb ${ }^{\mathrm{k}}$
}

\author{
${ }^{a}$ INGEBI/CADIC, CONICET, Vuelta de Obligado 2490, 1428 Buenos Aires, Argentina \\ ${ }^{b}$ Experimental Studies Division, Air Quality Research, Meteorological Service of Canada (ARQX), 4905 Dufferin \\ Street, M3H 5 T4 Downsview, Ontario, Canada \\ ${ }^{\circ}$ Code 613.3, NASA/Goddard Space Flight Centre, 20771 Greenbelt, MD, USA \\ ${ }^{d}$ WMO Secretariat, Chief, AER, RES, 7 bis, Avenue de la Paix, Case Postale No. 2300, CH-1211 Geneva 2, \\ Switzerland \\ ${ }^{e}$ Department of Physics, Faculty of Science, Silpakorn University, 73000 Nakhon Pathom, Thailand \\ ${ }^{f}$ Department of Physics, Norwegian University of Science and Technology, N-7491Trondheim, Norway \\ g Ozone Layer Monitoring Office, Japan Meteorological Agency, 1-3-4 Ote-machi, Chiyoda-ku, 100-8122 Tokyo, \\ Japan \\ ${ }^{h}$ Institute for Meteorology and Climatology, University of Hannover, Herrenhaeuser Str. 2, 30419 Hannover, \\ Germany \\ ${ }^{i}$ Institut d'Aéronomie Spatiale de Belgique, 3 avenue Circulaire, B-1180 Brussels, Belgium

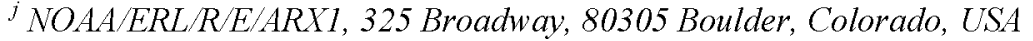 \\ ${ }^{k}$ School of Earth Atmospheric and Environmental Sciences, University of Manchester, Simon Building \\ M13 9PL Manchester, United Kingdom
}

\begin{abstract}
General objectives for measuring solar ultraviolet (UV) irradiance are: a) To establish a UV climatology by long-term monitoring, e.g. within a network, b) To detect trends in global UV irradiance, c) To provide datasets for specific process studies and for the validation of radiative transfer models and/or satellite derived UV irradiance at the Earth's surface, d) To understand geographic differences in global UV irradiance, e) To gain information about actual UV levels and their diurnal and seasonal variability, f) To provide data for public information and awareness (e.g. UV index). Solar ultraviolet radiation can be measured by different classes of instrument and it is crucial to match the instrument employed to the intended objective. It should also be ensured that sufficient facilities are available to support the objective in terms of personnel and QA/QC requirements. The three classes of instrument available for solar UV measurement are spectral, broadband and multifilter. Spectral instruments are the most costly, complex and demanding of those available, but provide the most versatile data. Broadband radiometers, tend to be cheaper and have fewer operational problems than spectroradiometers. However, their maintenance and QA/QC can introduce substantial additional cost. Multifilter radiometers combine some of the properties of both broadband and spectral instruments. Not all the above mentioned instruments are suited to all of the objectives. To achieve objectives a,d,e and $\mathrm{f}$, any class of instrument can be used, but it is necessary that it accomplishes a minimum requirement in quality. The second listed objective, trend detection, is the most demanding goal of UV monitoring, and spectral instruments are most suited to this task. The instrument specifications and the QA/QC requirements necessary to enable detection of small trends are very stringent and must be maintained over a prolonged period to justify trend detection. Providing datasets for process studies or satellite validation (objective $\mathrm{c}$ ) is again best served by spectral instruments. For the validation of radiative transfer models the accuracy of spectral measurements must be comparable to the accuracy needed for trend detection.
\end{abstract}

Keywords: Ultraviolet Radiation, Data Quality, Instruments.

PACS: 42.68.-w, 92.60.Ry, 92.70.Cp, 92.70.Gt, 92.70.Np, 93

CP1100, Current Problems in Atmospheric Radiation (IRS 2008) edited by T. Nakajima and M. A. Yamasoe

(1) 2009 American Institute of Physics 978-0-7354-0635-3/09/\$25.00 


\section{INTRODUCTION}

Solar ultraviolet (UV) radiation can be measured by several different classes of instrument and the data applied to a range of objectives. It is crucial to match the instrument employed to the intended objective, and to ensure that sufficient facilities are available to support the objective in terms of personnel and QA/QC requirements. The technical specifications of the different type of instrument for measuring solar UV radiation are detailed in the GAW series of publications "Instruments to measure solar ultraviolet radiation"(1-3), which should be read in conjunction with the Data Quality Objectives (DQOs) document (4). Quality control and quality assurance issues are covered in a further two GAW publications $(5,6)$.

The three classes of instrument available for solar UV measurement are spectral, broadband and multifilter. Spectral instruments provide high resolution spectral data that should cover the range $290-325 \mathrm{~nm}$ as a minimum, and ideally the full solar UV region $290-400 \mathrm{~nm}$. The spectral bandwidth should be less than $1 \mathrm{~nm}$ full width at half maximum, and tolerances on other aspects of instrument performance are detailed in (1). Spectral instruments are the most costly, complex and demanding of those available, but provide the most versatile data.

Broadband radiometers, for the purposes of this document, are taken as those instruments that measure the total erythemal (or "sunburning") irradiance (7) (type B-1). Deviations between the actual meter response and the true erythemal action spectrum can be accounted for in the calibration process (2). The UV index (8) is derived directly from the erythemal irradiance. The relative simplicity of broadband instruments means that they tend to be cheaper and have fewer operational problems than spectroradiometers. However, their maintenance and QA/QC can introduce substantial additional cost.

Multifilter radiometers combine some of the properties of both broadband and spectral instruments (3). They provide coarse spectral data by employing several different channels to detect radiation in a number of wavebands of typical width between 2 and $10 \mathrm{~nm}$. The spectral information available makes the data more versatile than that of a broadband radiometer, but they cannot match the detail and applications possible with a spectroradiometer. Routine operation, and QA/QC issues are a combination of those required for spectral and broadband instrument.

\section{OBJECTIVES}

General objectives for measuring solar ultraviolet irradiance are discussed below. There are numerous other research objectives that may require special consideration (eg a change of input optic), or that may use data gathered during routine monitoring for a new application, but here, only the objectives applicable to a global monitoring programme are discussed.

Objectives for monitoring solar UV radiation:

a) To establish a UV climatology by long-term monitoring, e.g. within a network

b) To detect trends in global UV irradiance

c) To provide datasets for specific process studies and for the validation of radiative transfer models and/or satellite derived UV irradiance at the Earth's surface

d) To understand geographic differences in global UV irradiance

e) To gain information about actual UV levels and their diurnal and seasonal variability.

f) To provide data for public information and awareness (e.g. UV index)

Not all instruments are suited to all of the objectives e.g. broadband instruments are not suited to trend detection unless the expected trends are large.

There are additional objectives that may be assigned to some instruments type deployed together with others, for example, for broadband (and multifilter) instruments installed attached to spectral instruments. His objectives are:

g) To supplement spectral UV measurements (e.g. temporal and spatial interpolation, interpretation of cloud effects).

h) To help in quality control of spectral UV measurements

\section{MATCHING OBJECTIVES AND INSTRUMENTS}

Objectives a),d),e) and f) above can be achieved using any class of instrument, but it is necessary to accomplish a minimum requirement in quality. Insights into the causes of geographical differences, diurnal or seasonal variability 
may be lost with a lack of spectral information, but a well maintained instrument of any type still provides valuable information about the UV climatology at a site.

Combining or comparing data from more than one site requires a greater emphasis to be placed on quality assurance (6) than when assessing a single site. Combining data from different instrument classes is possible at the loss of spectral resolution. Erythemal UV irradiance can be derived from both spectral and multifilter instruments, making this (and the UV index) a quantity common to all instruments. By corollary, the simpler broadband instruments can be used to help identify problems in the more complex spectral data that may otherwise go undetected (objective h).

The second listed objective, trend detection, is the most demanding goal of UV monitoring. Table (I) shows the requirements of spectral instruments to accomplish this objective. Even at a fixed site the incident UV is influenced by cloud, ozone, aerosol and albedo, all of which can vary tremendously from day to day and year to year, while being within normal ranges. Detecting a true trend, especially a small trend (all that is expected in most regions of the world) against such a background is extremely demanding. Any trend due to ozone depletion / recovery would be most obvious at the short wavelengths most affected by ozone, thus spectral instruments are most suited to this task. The instrument specifications (1) and the QA/QC requirements $(5,6)$ necessary to enable detection of small trends are also very stringent and must be maintained over a prolonged period to justify trend detection. A useful but ambitious goal is to attempt to detect a change in spectral UV irradiance resulting from a $1 \%$ change in total ozone column. In order to detect this change, the wavelength alignment accuracy must be significantly better than $\pm 0.05 \mathrm{~nm}$, the detection threshold must be in the order of $10-6 \mathrm{~W} \mathrm{~m}-2 \mathrm{~nm}-1$ or lower and the accuracy of the absolute calibration must be at least $\pm 5 \%$. (1). This objective is thus the most exacting and not easily achieved. Broadband radiometers might be suited to this task if large trends were to occur, or trends that were spectrally flat (eg due to changes in cloud cover). Otherwise, the role of the broadband radiometer in trend detection is found as a supporting instrument (objective h) in helping to maintain the tight quality control necessary for the task.

TABLE (I). Specifications spectral instruments type S-2 (1).

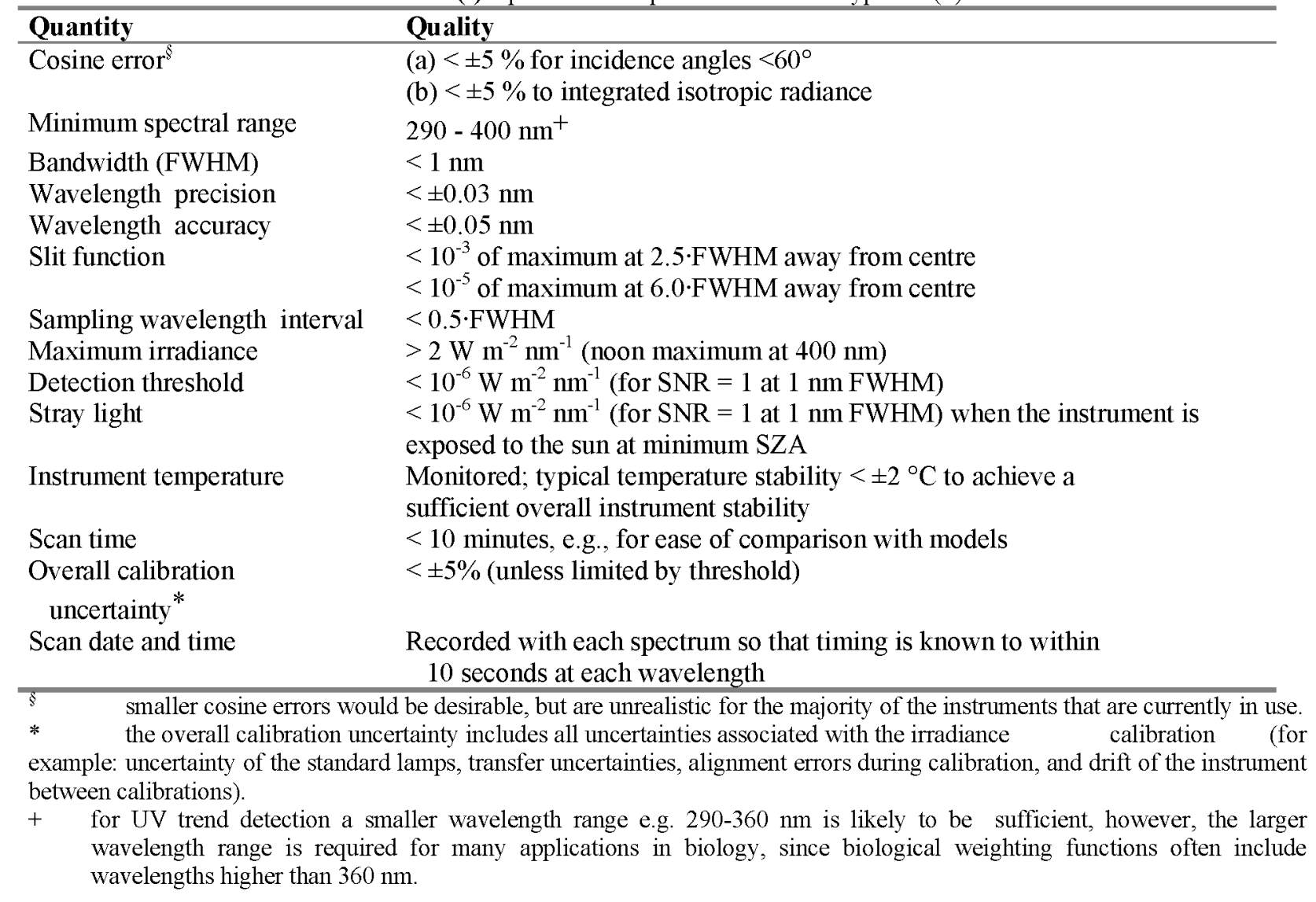


Providing datasets for process studies or satellite validation (objective c) is again best served by spectral instruments. For the validation of radiative transfer models the accuracy of spectral measurements must be comparable to the accuracy needed for trend detection (1).

The spectral data is necessary for identifying or disentangling processes, and provides the most stringent test of satellite retrievals. Nevertheless, there is also an important role for the radiometers in conjunction with the detailed spectral data. One of the disadvantages of spectral instruments is the time taken to make a measurement (for the scanning instruments: diode array and CCD devices do not have this problem, but as single monochromators they suffer from straylight problems in the UVB). They are also difficult to deploy in large numbers because of their demanding operational requirements. Thus the simpler radiometers with their rapid sampling capabilities and easier deployment can be used to help interpret the spectral data, or interpolate either spatially or temporally between spectra (objective g).

For all the objetives, data collection needs to be automated and all weather. One scan per hour, plus one scan at noon, is the minimum required data frequency for most of the objectives. For objective b) (trends detection), the measurement schedule should comprise sufficient scans to enable an accurate ( $\pm 10 \%$ uncertainty) daily integral of global spectral irradiance (i.e., daily global spectral irradiation) for days with no rain.

In order to guarantee a good quality data set, instruments should be calibrated at least once a year, and all calibration information and procedures should be clearly documented and archived at the observation site. In addition, daily, weekly and monthly tasks need to be accomplished, according to the instrument type and objective.

Further specifications for, and justifications of, the instrument requirements for each objective are provided in the Instrument document series (1-3).

\section{ACKNOWLEDGMENTS}

The authors want to thank the AREP (WMO) and their own institutions for their support to produce this document.

\section{REFERENCES}

1. Instruments to measure solar ultraviolet radiation. Part 1: Spectral instruments. WMO/GAW report 125 (2001)

2. Instruments to measure solar ultraviolet radiation. Part 2: Broadband instruments measuring erythemally weighted broadband irradiance. WMO/GAW report xxx (in press).

3. Instruments to measure solar ultraviolet radiation. Part 3: Multifilter instruments. WMO/GAW report yyy (in preparation).

4. Data Quality Objectives (DQO) for Solar Ultraviolet Radiation, WMO/GAW report (in preparation.

5. Guidelines for site quality control of UV monitoring. WMO/GAW report 126 (1998).

6. Quality assurance in monitoring solar ultraviolet radiation: the state of the art. WMO/GAW report 146 (2003).

7. MacKinley, A. F. and Diffey, B.L. (1987) A reference action spectrum for ultraviolet induced erythema in human skin, CIE J., $6(1), 17-22$

8. WHO (World Health Organisation) (2002) Global Solar UV Index. A Practical Guide. WHO, Geneva, Switzerland. 